\title{
Computed tomography appearances in paediatric tuberous sclerosis
}

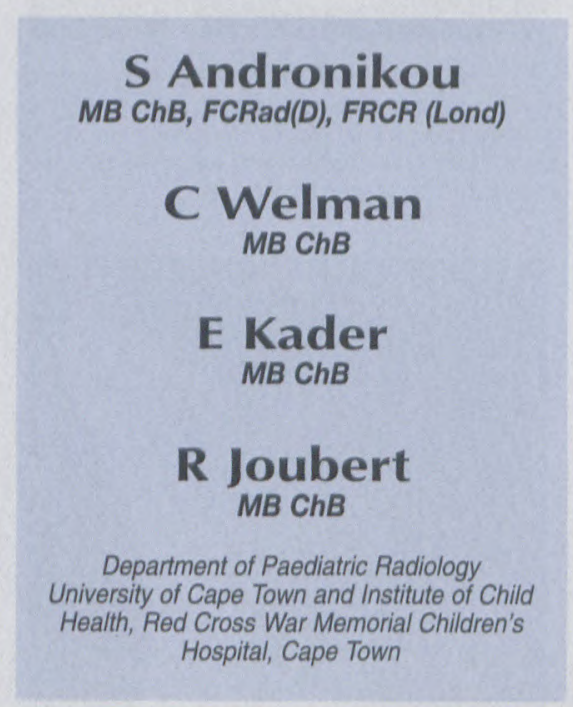

Identification of one or more of three characteristic imaging findings may make the radiological diagnosis of tuberous sclerosis in childhood. The computed tomography (CT) scan appearances vary according to age and it is important that the radiologist be aware of this, so as to make the correct diagnosis.

\section{Subependymal hamartomas}

These are noted on the ventricular surface of the caudate nucleus. Less common sites include the frontal and temporal portions of the lateral ventricles as well as around the 3rd and 4 th ventricles. These lesions change with patient age - in the first year of life they are rarely calcified, but after 1 year of age they calcify commonly. Variable contrast enhancement is noted, ranging from not at all to marked enhancement. Giant cell tumour is an enlarging subependymal nodule at the foramen of Monro and is recognised by its location and evidence of growth on serial studies. These only rarely degenerate into high-grade infiltrating lesions, when they are seen to invade parenchyma or show rapid enlargement.
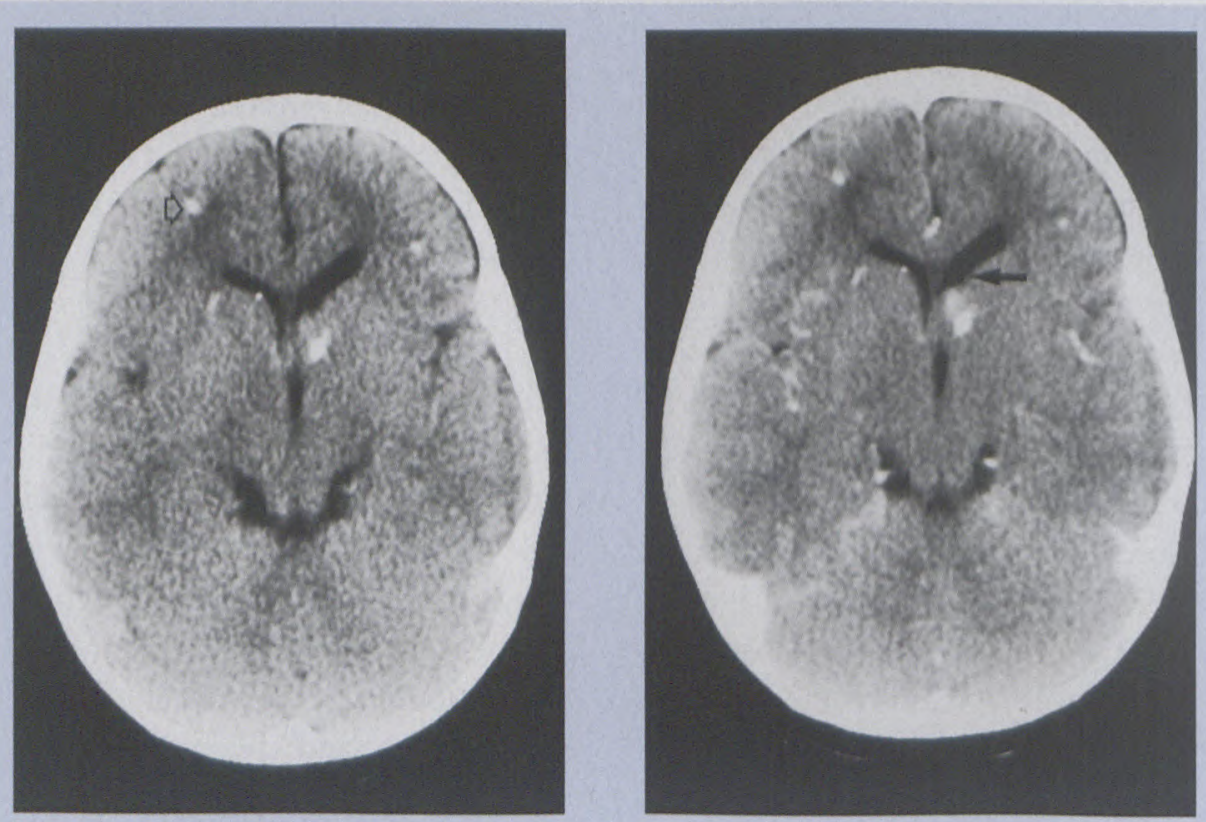

Figs $2 \mathrm{a}$ and $2 \mathrm{~b}$. Pre and post-contrast CT scans show a lett-sided, partially calcified subependymal nodule at the foramen of Monro, which shows marked contrast enhancement (closed arrows). The open arrows indicate white matter lesions of low density with partial calcification (high-density focus). These do not enhance with con-

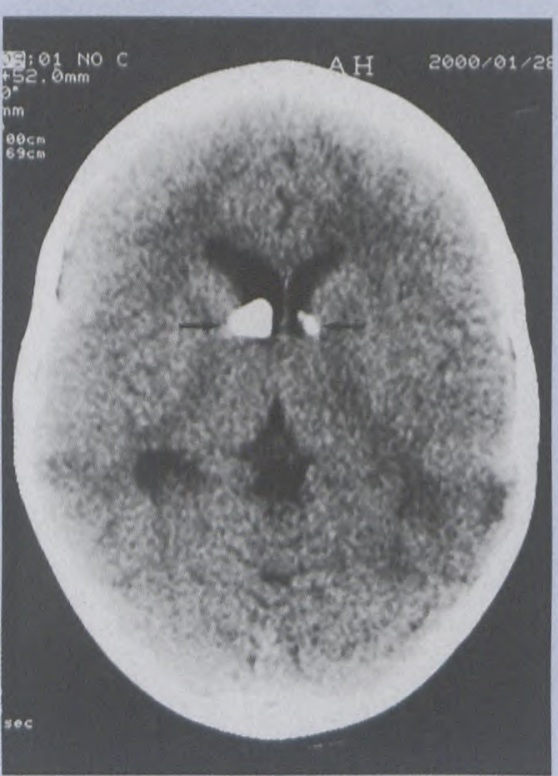

Fig. 1. Bilateral calcified subependymal hamartomas at the foraminae of Monro (arrows) in an older child. These lesions may also occlude the foraminae resulting in hydrocephalus.

\section{Cortical hamar- tomas/tubers}

Calcification of these lesions increases with increasing age. They may be gyriform simulating Sturgetrast. 


\section{CLINICAL IMAGES}

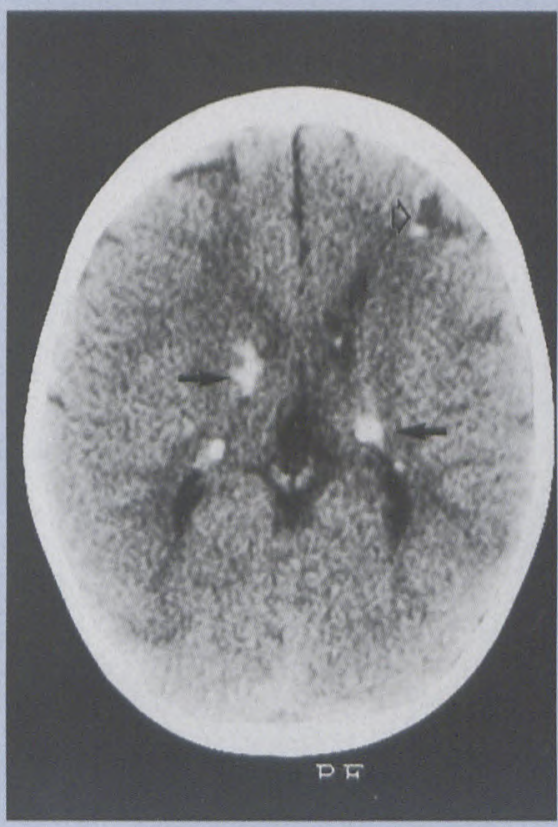

Fig. 3. Subependymal calcified hamatomas (arrows) are seen along the surface of the bodies of the lateral ventricles. A cortical tuber, which is partially calcified, is also present (open arrow).

Weber syndrome. These do not usually enhance (unlike subependymal nodules) unless they are degenerating. They also vary in appearance with age

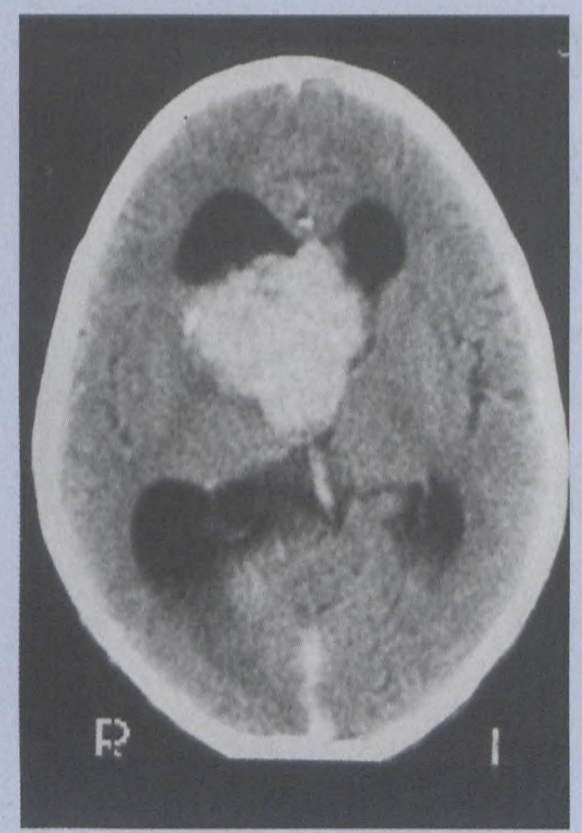

Fig. 4. A large enhancing midline mass at the foramen of Monro developed at the site of previously identified harmatomas. This is now termed a giant cell astrocytoma.

- below 1 year of age they are of low density and are associated with broad gyri. With increasing age the density increases, until adulthood where dif- ferentiation from normal cortex can be difficult unless they are calcified.

\section{White matter lesions}

These are low-density well-defined areas in the cerebral white matter which do not enhance but can calcify.'

MRI is more sensitive in identifying all the above lesions. Varying signal intensities on $\mathrm{T} 1, \mathrm{~T} 2$ and gadolinium-enhanced studies are noted according to the patient's age. MRI also shows characteristic curvilinear high T2 signal intensity extensions from subependymal hamartomas to cortical tumours which represent unmyelinated, disordered cells along the path of the glial-neuronal units (Figs 2-4).

\section{Reference}

1. Barkovich AJ. Paediatric Neuroimaging. 2nd ed. Philadelphia: Lippincott-Raven, 1996: 296-304. 